\title{
Review of: "Radiation therapy-associated toxicity: Etiology, management, and prevention"
}

\author{
Alexandros Georgakilas ${ }^{1}$ \\ 1 National Technical University of Athens
}

Potential competing interests: The author(s) declared that no potential competing interests exist.

In this extensive review, Wang and Tepper as clinicians, approach the important topic or radiation therapy (RT)- associated toxicity in a nice and relatively inclusive manner but with emphasis on the clinical and not mechanistic aspects of normal tissue toxicity for patients undergoing RT (etiology). Nevertheless, the review is well-organized and covers the full range of side-effects (acute and late) in different organs and tissues from skin, breast, prostate to central nervous system and head and neck etc. The problem with radiation toxicity is that many times clinicians and basic science scientists do not consider this issue in a systems biology approach simulating the human organism. This review approaches the topic rather weak as far as it concerns the following issues:

1. The authors could have discussed in a more analytical way, the toxicities (Table 1 ) occuring after which specific type of radiation therapy and protocol i.e. more specific clinical aspects for each tumor type treatment

2. An aspect that is totall missing from the current review, is the systemic nature of radiation effects. It is currently more and more embraced by the scientific community, that the etiology for ionizing radiation side effects and toxicity documented in animals and humans involves some primary and secondary mechanims starting from the induction of complex and dense (clustered) DNA damage and biological cellular/tissue damage in general (see Mavragani, I.V. et al. (2019) Ionizing Radiation and Complex DNA Damage: From Prediction to Detection Challenges and Biological Significance. Cancers, 11, 1789). This induction of extensive and dense damage are highly repair resistant leading to a genuine stress oftern referred to as radiation stress and the triggering of a series of systemic effects effecting not only nearby cells and tissues (bystander) but also distant. This complex phenomenon is definitely involved in the exhibition of toxicity but also the so-called 'abscopal' effect which may have also positive role in tumor control (Rodriguez-Ruiz et al. M.E., Vanpouille-Box, C., Melero, I., Formenti, S.C. and Demaria, S. (2018) Immunological Mechanisms Responsible for Radiation-Induced Abscopal Effect. Trends Immunol, 39, 644-655).

3. The authors in addition to the above missing point, could have included the upcoming idea of damageassociated molecular patterns (DAMPs) often acting as danger signals in many cases of biological damage occuring by radiation, oxidative or even reperfusion injury stress (Heil, M. and Land, W.G. (2014) Danger signals - damaged-self recognition across the tree of life. Frontiers in plant science, 5; 
Land, W.G. (2018) Damage-Associated Molecular Patterns in Human Diseases. Volume 1: Injury-Induced Innate Immune Responses. Springer International Publishing AG, part of Springer Nature 2018).

4. The reader could have benefit by adding information on the role of dose in normal tissues as weakly discussed in Figure 3 .

5. The type of radiation therapy X-rays or protons/carbons should have incorporated in the analysis of different toxicities. 